\title{
Power Optimization for Aerial Intelligent Reflecting Surface-Aided Cell-Free Massive MIMO-Based Wireless Sensor Network
}

\author{
Tao Zhou $\mathbb{D}^{1},{ }^{1}$ Kui Xu $\mathbb{D}^{\mathbb{D}},{ }^{1}$ Chunguo $\mathrm{Li} \mathbb{D}^{\mathrm{D}},{ }^{2}$ and Zhexian Shen $\mathbb{D}^{1}$ \\ ${ }^{1}$ The College of Communications Engineering, Army Engineering University of PLA, Nanjing, China \\ ${ }^{2}$ Southeast University, Nanjing, China \\ Correspondence should be addressed to Kui Xu; lgdxxukui@sina.com
}

Received 26 July 2021; Revised 15 August 2021; Accepted 23 August 2021; Published 25 September 2021

Academic Editor: Xin Liu

Copyright (c) 2021 Tao Zhou et al. This is an open access article distributed under the Creative Commons Attribution License, which permits unrestricted use, distribution, and reproduction in any medium, provided the original work is properly cited.

\begin{abstract}
Intelligent reflecting surfaces (IRSs) have significant advantages in enhancing the coverage and reducing the deployment cost of wireless networks. This paper studies an aerial IRS- (AIRS-) enhanced cell-free massive multiple-input multiple-output- (MIMO-) based wireless sensor network (WSN) in which multiple access points (APs) serve several sensor users (SUs). Direct links between the APs and SUs are blocked due to occlusion by tall buildings. Hence, we deploy an AIRS to improve the communication quality of the SUs. Our goal is to minimize the total transmit power of all APs under a given minimum signal-to-interference-plus-noise ratio (SINR) requirement. We propose a joint iterative optimization algorithm by designing an active beamforming mechanism at each AP and a passive beamforming mechanism at the AIRS to solve this problem. Simulation results illustrate the good performance of the proposed method.
\end{abstract}

\section{Introduction}

Compared with fifth-generation (5G) communication, sixth-generation $(6 \mathrm{G})$ communication will require higher speeds, lower latency, and Internet of Everything (IoE) capabilities [1-3]. In addition, $6 \mathrm{G}$ will achieve integrated airspace-ground coverage, providing safe and reliable service [4-6]. As one of the core technologies for 6G, intelligent reflective surfaces (IRSs) can effectively enhance the regional coverage and reduce the energy consumption of wireless networks [7]. Therefore, IRSs have received widespread attention in recent years.

An IRS consists of many programmable subwavelength metamaterial array elements. Each element can control the amplitude and phase of electromagnetic waves to realize smart and reconfigurable transmission based on the wireless environment [8-10]. In contrast to traditional transmitters, an IRS does not perform any signal processing and merely modifies the phase and amplitude of incident electromagnetic waves. An IRS does not need to be equipped with a radio frequency link composed of filters, mixers, and power amplifiers. Therefore, IRSs can effectively reduce the costs and energy consumption of wireless networks $[11,12]$. The signals reflected by an IRS can be made to be coherently superimposed at the receiver by suitably designing the reflection coefficients of the IRS. We call this approach passive beamforming $[13,14]$.

Cell-free (CF) massive multiple-input multiple-output (MIMO) technology breaks away from the fixed architecture of traditional cellular massive MIMO. In a CF system, multiple distributed access points (APs) provide communication services for several users in the coverage area simultaneously. All the APs are connected through backhaul links and are controlled by a smart central processing unit (CPU). The CPU can control the resource allocation in real time, thereby eliminating the cell boundaries in traditional cellular networks. Thus, the interference from different cells can be expunged [15-18].

To enhance the coverage of a wireless network, a large number of distributed APs need to be deployed. However, 
limited by their deployment cost and energy consumption, high-density deployment of APs is impractical. Based on the above analysis, an IRS can be deployed in a CF massive MIMO network to enhance the communication quality. The deployed IRS can replace some APs in providing services for users. Due to its low cost and flexible deployment characteristics, the IRS can enhance the coverage of the CF massive MIMO system while reducing its deployment cost. However, due to the complex distribution of urban buildings, the deployment of an IRS (which is usually located on a wall or roof) depends on the buildings that are present in the area. Some of the best IRS deployment locations may not be suitable in practice due to topographical or architectural constraints. Therefore, to overcome the terrain limitations of traditional ground IRS deployment and make full use of airground resources, an aerial IRS (AIRS) was proposed as a new IRS architecture in $[19,20]$. An AIRS is usually installed on a launch platform (a launch balloon or unmanned aerial vehicle, UAV) to establish a strong line-of-sight (LoS) connection. The AIRS location can then be adjusted in accordance with current service needs, in line with the usercentred principle.

In $6 \mathrm{G}$ communication, a large number of intelligent devices will not only provide convenience for our life but also constitute a huge wireless sensor network (WSN). The WSN requires higher transmission rate. To solve this problem, we can use the IRS to increase coverage, improve spectrum efficiency, and reduce the deployment cost of wireless networks.

Motivations and Contributions. The current research on IRSs has mainly focused on traditional cellular networks, including IRS-assisted multiple-input single-output (MISO) communication [13, 21, 22], MIMO communication [23], and multicell interference cancellation scenarios [24]. There have been only a few studies on the application of IRSs in CF massive MIMO networks, mainly focusing on the rate optimization problem [25-28], while the problem of power minimization has not been addressed. In $[13,22]$, the problem of power optimization for single-IRS-assisted single-cell communication was studied. However, power optimization for an IRS-aided CF massive MIMO network has not yet been investigated. In addition, the existing research mainly focuses on the single-IRS case. There are a few studies that consider the multi-IRS case. For the multi-IRS case, the IRS deployment strategy is important in enhancing the communication quality. However, few studies consider the IRS deployment strategy. Moreover, the power optimization problem in the IRS-aided WSN system has not been considered yet. Therefore, in this study, we consider the AIRS-enhanced CF massive MIMO WSN system. The main contributions are summarized as follows:

(1) In this paper, we first consider the power optimization problem for the AIRS-aided CF massive MIMO-based WSN system. Several APs serve several sensor users (SUs) with the assistance of an AIRS. This paper aims to minimize the total transmit power of all APs under a signal-to-interference-plus-noise ratio (SINR) requirement.

(2) We propose a joint optimization algorithm to optimize the total transmission power for the singleAIRS case. Then, we extend to power optimization for the multi-AIRS case. The simulation results show the good performance of our proposed method in power savings.

(3) Moreover, we discuss the AIRS deployment strategy. Compared with centralized AIRS deployment, distributed AIRS deployment shows better performance, providing some guidelines for AIRS deployment.

1.1. Methods. Based on the idea of alternating iterations commonly used in IRS-assisted transmission optimization, we jointly optimize the beamforming vectors and AIRS reflection coefficients to solve the transmission power optimization problem. Specifically, the optimization problem is divided into two subproblems: fixing the AIRS reflection coefficients to optimize the beamforming vectors and fixing the beamforming vectors to optimize the AIRS reflection coefficients. We can obtain an optimal solution by iteratively optimizing the above two subproblems. The transmission power can be effectively reduced by using the proposed method.

1.2. Organization. In Section 2, we introduce and analyze the system model and formulate the power optimization problem. We use the Ricean channel model to characterize the air-to-ground channels. In Section 3, we introduce our proposed optimization strategy and analyze it in detail. In Section 4, we present a numerical simulation performed to verify the performance of the proposed method. Finally, in Section 5, we summarize this paper, analyze the deficiencies of this work, and then identify future research directions.

1.3. Notations. We use italic letters for constants $(B)$ and bold lowercase letters to denote vectors (p). Matrices are represented by bold capital letters. The argument of a complex number $B$ is denoted by $\arg (B)$. For a vector $\mathbf{p}$, we use $\arg (\mathbf{p})$ to express its corresponding argument vector, and $\operatorname{diag}(\mathbf{p})$ denotes a diagonal matrix whose diagonal elements are the elements in $\mathbf{p} . \mathbf{A}^{H}$ denotes the conjugate transpose of a matrix $\mathbf{A}$, and $\mathbf{A}^{T}$ is the transpose of a matrix A. $\otimes$ is the Kronecker product.

\section{System Model and Problem Formulation}

As shown in Figure 1, in the considered CF massive MIMObased WSN system, $L$ multiantenna APs serve $K$ singleantenna SUs. Tall buildings block direct links between the APs and SUs. An AIRS is deployed to enhance the quality of service (QoS) of the SUs. Suppose that each AP has the form of a uniform linear array (ULA), while the AIRS has the form of an $N_{x} \times N_{y}$ uniform plane array. Each AP has $M$ 


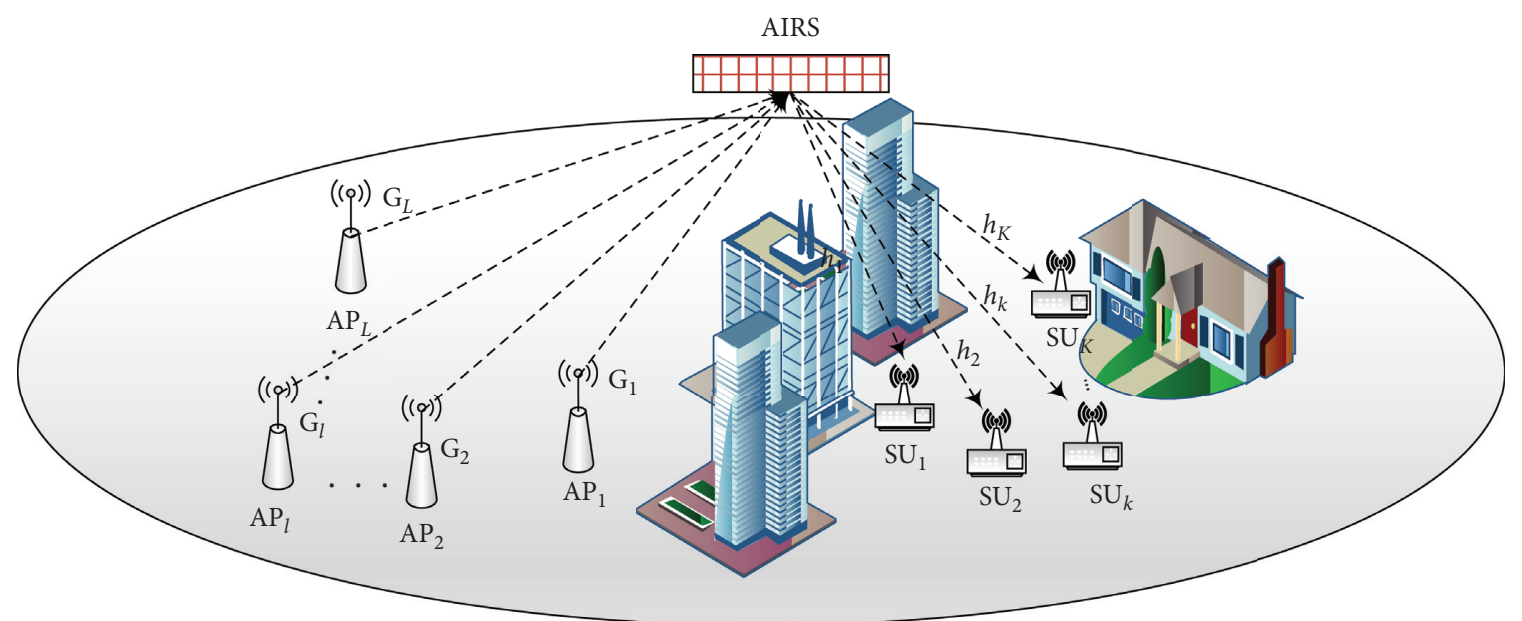

Figure 1: System model.

antennas. The AIRS consists of $N=N_{x} \times N_{y}$ elements, with half-wavelength spacing of the antennas/elements. Similar to $[13,22,29-33]$, we characterize the channel from the $l$-th AP to the AIRS $\mathbf{G}_{l} \in \mathbb{C}^{N \times M}$ as follows:

$$
\mathbf{G}_{l}=\sqrt{\beta_{\mathbf{G}_{l}}}\left(\sqrt{\frac{\kappa}{\kappa+1}} \psi_{N}\left(\theta_{i}, \varphi_{i}\right) \varsigma_{M}^{\mathrm{H}}\left(\theta_{i}\right)+\sqrt{\frac{1}{\kappa+1}} \widetilde{\mathbf{G}}_{l}\right),
$$

where $\widetilde{\mathbf{G}}_{l}$ denotes the non-line-of-sight (NLoS) component, whose elements are chosen from $\mathscr{C} \mathscr{N}(0,1) \cdot \beta_{\mathbf{G}_{l}}$ is the path loss. Here, we define $\beta_{\mathbf{G}_{l}}$ as

$$
\beta_{\mathbf{G}_{l}}=\frac{a_{0}}{d_{\mathbf{G}_{l}}^{2}}
$$

where $a_{0}$ is the path loss at a reference distance of $1 \mathrm{~m}$ and $d_{\mathbf{G}_{l}}$ is the distance between the $l$-th AP and the AIRS. $\psi_{N}\left(\theta_{i}, \varphi_{i}\right)$ and $\varsigma_{M}\left(\theta_{i}\right)$ are string vectors that can be expressed as

$$
\psi_{N}\left(\theta_{i}, \varphi_{i}\right)=\left[1, e^{-j \pi \tau \sin \left(\theta_{i}\right) \sin (\varphi)}, \ldots, e^{-j \pi \tau\left(N_{x}-1\right) \sin \left(\theta_{i}\right) \sin \left(\varphi_{i}\right)}\right]^{T} \otimes\left[1, e^{-j \pi \tau \sin \left(\theta_{i}\right) \cos \left(\varphi_{i}\right)}, \ldots, e^{-j \pi \tau\left(N_{y}-1\right) \sin \left(\theta_{i}\right) \cos \left(\varphi_{i}\right)}\right]^{T}
$$

And

$$
\varsigma_{M}\left(\theta_{i}\right)=\left[1, e^{j \pi \tau \cos \left(\theta_{i}\right)}, \ldots, e^{j \pi \tau(M-1) \cos \left(\theta_{i}\right)}\right]^{T} .
$$

When $\kappa=0$, the above model reduces to the Rayleigh fading channel model. An AIRS is usually deployed at a high altitude. From an elevated position, the AIRS can more easily establish LoS links with the ground nodes, which leads to LoS-dominated channel conditions $[19,20,34,35]$. Similarly, the channel from the AIRS to the $k$-th SU can be modeled as

$$
\mathbf{h}_{k}=\sqrt{\beta_{\mathbf{h}_{k}}}\left(\sqrt{\frac{\kappa}{\kappa+1}} \psi_{N}\left(\alpha_{k}, \eta_{k}\right)+\sqrt{\frac{1}{\kappa+1}} \widetilde{\mathbf{h}}_{k}\right) .
$$

$\mathbf{h}_{k} \in \mathbb{C}^{1 \times N}$. The corresponding symbols are defined similarly to the case of $\mathbf{G}_{l}$.
All APs serve SUs simultaneously. The received signal at the $k$-th SU can be expressed as

$$
\begin{aligned}
y_{k} & =\sum_{l=1}^{L} \mathbf{c}_{l k} \mathbf{x}_{l}+\varpi_{k} \\
& =\sum_{l=1}^{L} \mathbf{c}_{l k} \sum_{i=1}^{K} \mathbf{w}_{l i} s_{i}+\varpi_{k} \\
& =\sum_{l=1}^{L} \mathbf{c}_{l k} \mathbf{w}_{l k} s_{k}+\sum_{l=1}^{L} \mathbf{c}_{l k} \sum_{i \neq k}^{K} \mathbf{w}_{l i} s_{i}+\varpi_{k},
\end{aligned}
$$

where $\mathbf{x}_{l}$ denotes the transmit signal of the $l$-th AP, and $\mathbf{x}_{l}=\sum_{i=1}^{K} \mathbf{w}_{l i} s_{i} ; \mathbf{w}_{l k}$ is the beamforming vector at the $l$-th AP to the $k$-th SU; $s_{k}$ is the information symbol sent to the $k$-th $\mathrm{SU} ; \Phi_{k}$ is the additive white Gaussian noise (AWGN) at the $k$-th SU, and $\omega_{k} \sim \mathscr{C} \mathcal{N}\left(0, \sigma^{2}\right)$; and $\mathbf{c}_{l k} \in \mathbb{C}^{1 \times M}$ is the AIRS- 
reflected channel between the $l$-th AP and the $k$-th SU, which can be expressed as

$$
\begin{aligned}
\mathbf{c}_{l i} & =\mathbf{h}_{i} \boldsymbol{\Phi} \mathbf{G}_{l} \\
& =\mathbf{v}^{H} \operatorname{diag}\left(\mathbf{h}_{i}\right) \mathbf{G}_{l} \\
& =\mathbf{v}^{H} \mathbf{U}_{l i},
\end{aligned}
$$

where $\boldsymbol{\Phi}=\operatorname{diag}\left(e^{j \omega_{1}}, e^{j \omega_{2}}, \ldots, e^{j \omega_{N}}\right) \in \mathbb{C}^{N \times N}$ is the reflection coefficient matrix of the AIRS, $\omega_{n} \in[0,2 \pi)$ is the reflection coefficient of the $n$-th element, and $\mathbf{v}=\left[e^{j \omega_{1}}, e^{j \omega_{2}}\right.$, $\left.\ldots, e^{j \omega_{N}}\right]^{H}$. The SINR of the $k$-th SU can be expressed as

$$
\operatorname{SINR}_{k}=\frac{\left|\sum_{l=1}^{L} \mathbf{c}_{l k} \mathbf{w}_{l k}\right|^{2}}{\sum_{i \neq k}^{K}\left|\sum_{l=1}^{L} \mathbf{c}_{l i} \mathbf{w}_{l i}\right|^{2}+\sigma^{2}} .
$$

In this paper, we aim to minimize the transmission power $P$ of all APs, $P=\sum_{l=1}^{L} \sum_{i=1}^{K}\left\|\mathbf{w}_{l}\right\|^{2}$. Thus, the problem to be optimized is constructed as follows:

$$
\begin{aligned}
& P 1: \\
& \min _{\mathbf{v}, \mathbf{w}_{l i}} \sum_{l=1}^{L} \sum_{i=1}^{K}\left\|\mathbf{w}_{l i}\right\|^{2}, \\
& \text { s.t. } \\
& \quad \operatorname{SINR}_{k} \geq \gamma_{k}, \quad k=1,2, \ldots, K, \\
& \quad \omega_{n} \in[0,2 \pi), \quad n=1,2, \ldots, N .
\end{aligned}
$$

\section{Joint Beamforming and Reflection Design}

To solve $(P 1)$, we need to design the beamforming mechanism at each AP and the AIRS reflection coefficients. As we can see from (7) and (9), all channels $\mathbf{c}_{l k}(l=1, \ldots, L$, $k=1, \ldots, K)$ vary with $\boldsymbol{\Phi}$. If we modify the AIRS reflection coefficients, the corresponding beamforming design for the APs will also change. Therefore, it is difficult to solve $(P 1)$. Inspired by the traditional idea of alternating iterations in IRS-aided communication [22, 33], we propose a joint optimization strategy to solve this problem. That is, first, for fixed $\Phi$, we optimize the beamforming at the APs, and then, for fixed beamforming design at the APs, that is, $\mathbf{w}_{l i}$, we optimize the passive beamforming at the AIRS. The above steps are alternatively performed until convergence.

3.1. Beamforming Design for the APs. Note that when the AIRS reflection coefficients $\Phi$ are fixed, we need only to optimize $\mathbf{w}_{l i}$ to minimize $P$. Then, $(P 1)$ can be simplified as follows:

$$
\begin{array}{ll}
\text { P2: } & \\
\min _{\mathbf{w}_{l i}} & \sum_{l=1}^{L} \sum_{i=1}^{K}\left\|\mathbf{w}_{l i}\right\|^{2}, \\
\text { s.t. } & \operatorname{SINR}_{k} \geq \gamma_{k}, \quad k=1,2, \ldots, K .
\end{array}
$$

In accordance with (8), we define $\mathbf{c}_{k}=\left[\mathbf{c}_{1 k}, \mathbf{c}_{2 k}, \ldots, \mathbf{c}_{L k}\right]$ and $\mathbf{w}_{k}=\left[\begin{array}{llll}\mathbf{w}_{1 k} & \mathbf{w}_{2 k} & \cdots & \mathbf{w}_{L k}\end{array}\right]^{\mathrm{T}} . \mathbf{c}_{k} \in \mathbb{C}^{1 \times M L}$ is the reflected channel vector of the $k$-th $\mathrm{SU}$, and $\mathbf{w}_{k} \in \mathbb{C}^{M L \times 1}$ is the beamforming vector for the $k$-th SU. Thus, we have $\sum_{l=1}^{L} \mathbf{c}_{l i} \mathbf{w}_{l j}=\mathbf{c}_{i} \mathbf{w}_{j}$. Therefore, (P2) can be converted into

P3:

$$
\min _{\mathbf{w}_{i}} \sum_{i=1}^{K}\left\|\mathbf{w}_{i}\right\|^{2}
$$

$$
\text { s.t. } \quad \frac{\left|\mathbf{c}_{k} \mathbf{w}_{k}\right|^{2}}{\sum_{i \neq k}^{K}\left|\mathbf{c}_{k} \mathbf{w}_{i}\right|^{2}+\sigma^{2}} \geq \gamma_{0}, \quad k=1,2, \ldots, K .
$$

From (11), we can find that $(P 3)$ is a convex problem when $\Phi$ is fixed. We can use a semidefinite programming(SDP-) based method to solve this problem. Note that

$$
\begin{aligned}
\left|\mathbf{c}_{k} \mathbf{w}_{k}\right|^{2} & =\mathbf{c}_{k} \mathbf{w}_{k}\left(\mathbf{c}_{k} \mathbf{w}_{k}\right)^{H} \\
& =\operatorname{Tr}\left(\mathbf{c}_{k} \mathbf{c}_{k}^{H} \mathbf{w}_{k} \mathbf{w}_{k}^{H}\right) \\
& =\operatorname{Tr}\left(\mathbf{C}_{k} \mathbf{W}_{k}\right),
\end{aligned}
$$

where $\mathbf{C}_{k}=\mathbf{c}_{k} \mathbf{c}_{k}^{H}$ and $\mathbf{W}_{k}=\mathbf{w}_{k} \mathbf{w}_{k}^{H}$. Then, (P3) can be considered to be equivalent to

P4:

$$
\begin{array}{ll}
\min _{w_{l i}} & \sum_{i=1}^{K} \operatorname{tr}\left(\mathbf{W}_{i}\right), \\
\text { s.t. } & \operatorname{tr}\left(\mathbf{C}_{k} \mathbf{W}_{k}\right) \geq \gamma_{0}\left(\sum_{i \neq k}^{K} \operatorname{tr}\left(\mathbf{C}_{k} \mathbf{W}_{i}\right)+\sigma^{2}\right), \quad k=1,2, \ldots, K, \\
& \mathbf{W}_{k} \succcurlyeq 0, \quad k=1,2, \ldots, K .
\end{array}
$$

$(P 4)$ is an SDP problem, and $\mathbf{W}_{k}$ can be solved by using a convex optimizer such as CVX [36]. Similar to [37], $\mathbf{W}_{k}$ satisfies the rank-one property, and the optimal beamforming design $\mathbf{w}_{k}^{\text {opt }}$ can be obtained via the rank-one decomposition (eigenvalue decomposition) of $\mathbf{W}_{k}$.

3.2. Reflection Design of the AIRS. When the beamforming design is determined, we can optimize $\Phi$ to further reduce the total transmission power. When the beamforming vectors $\mathbf{w}_{k}(k=1, \ldots, K)$ are fixed, the objective function can be rewritten as follows:

$$
\begin{array}{ll}
\text { P5: } & \lim _{x \longrightarrow \infty} \\
\max _{\Phi} & \sum_{i=1}^{K}\left\|\mathbf{w}_{l i}\right\|^{2}, \\
\text { s.t. } & \frac{\left|\sum_{l=1}^{L} \mathbf{c}_{l k} \mathbf{w}_{l k}\right|^{2}}{\sum_{i \neq k}^{K}\left|\sum_{l=1}^{L} \mathbf{c}_{l k} \mathbf{w}_{l i}\right|^{2}+\sigma^{2}} \geqslant \gamma_{0}, \quad k=1,2, \ldots, K,
\end{array}
$$$$
\omega_{n} \in[0,2 \pi), \quad n=1, \ldots, N .
$$ 
According to (7), $\mathbf{c}_{l i}=\mathbf{v}^{H} \mathbf{U}_{l i}$, where $\mathbf{U}_{l i}=\operatorname{diag}\left(\mathbf{h}_{i}\right) \mathbf{G}_{l}$. Then, the SINR constraint in (14) can be written as

$$
\frac{\left|\sum_{l=1}^{L} \mathbf{v}^{\mathrm{H}} \mathbf{U}_{l k} \mathbf{w}_{l k}\right|^{2}}{\sum_{i \neq k}^{K}\left|\sum_{l=1}^{L} \mathbf{v}^{H} \mathbf{U}_{l k} \mathbf{w}_{l i}\right|^{2}+\sigma^{2}} \geqslant \gamma_{0} .
$$

Here, we define $\mathbf{U}_{k}=\left[\mathbf{U}_{1 k}, \mathbf{U}_{2 k}, \ldots, \mathbf{U}_{L k}\right] \in \mathbb{C}^{N \times M L}$. Then, (15) can be written as

$$
\frac{\left|\sum_{l=1}^{L} \mathbf{v}^{H} \mathbf{U}_{k} \mathbf{w}_{k}\right|^{2}}{\sum_{i \neq k}^{K}\left|\sum_{l=1}^{L} \mathbf{v}^{\mathrm{H}} \mathbf{U}_{k} \mathbf{w}_{i}\right|^{2}+\sigma^{2}} \geqslant \gamma_{0} .
$$

$(P 5)$ can be expressed as

P6:

$$
\begin{aligned}
\max _{\mathbf{v}} & \sum_{k=1}^{K}\left\|\mathbf{w}_{k}\right\|^{2}, \\
\text { s.t. } & \frac{\left|\mathbf{v}^{H} \mathbf{U}_{k} \mathbf{w}_{k}\right|^{2}}{\sum_{i \neq k}^{K}\left|\mathbf{v}^{H} \mathbf{U}_{k} \mathbf{w}_{i}\right|^{2}+\sigma^{2}} \geqslant \gamma_{0}, \quad k=1,2, \ldots, K, \\
& \omega_{n} \in[0,2 \pi), \quad n=1, \ldots, N .
\end{aligned}
$$

Note that (P6) is not a convex problem with a unitmodulus constraint $\omega_{n} \in[0,2 \pi), n=1, \ldots, N$. Moreover, the objective function does not contain $\mathbf{v}$. To solve (P6), we can only find a vector $\mathbf{v}$ to satisfy the constraints in (17). Therefore, it is difficult to solve $(P 6)$. Instead, we will rewrite $(P 6)$ as an equivalent optimization problem and relax (P6) into an SDP problem to obtain an optimal solution.

When the beamforming vectors are obtained, the current setting of $\mathbf{v}$ already satisfies the SINR constraint condition. In other words, the SINR of each SU satisfies $\operatorname{SINR}_{k} \geqslant \gamma_{0}$. To reduce the total transmission power, we need to find a vector $\mathbf{v}^{*}$ such that the new SINR constraints calculated with $\mathbf{v}^{*}$ satisfy the following conditions:

$$
\begin{aligned}
\operatorname{SINR}_{k}^{*} & \geqslant \gamma_{0}^{*}, \quad k=1,2, \ldots, K, \\
\gamma_{0}^{*} & \geqslant \gamma_{0} .
\end{aligned}
$$

$\mathbf{v}^{*}$ leads to a higher SINR threshold value of $\gamma_{0}^{*}$. Thus, the transmission power can be reduced. From (17), we can find that, for the $k$-th SU, $\left|\mathbf{v}^{H} \mathbf{U}_{k} \mathbf{w}_{k}\right|^{2}$ is the useful component, while $\sum_{i \neq k}^{K}\left|\mathbf{v}^{\mathrm{H}} \mathbf{U}_{k} \mathbf{w}_{i}\right|+\sigma^{2}$ is the interference-plus-noise component. Define the SINR margin of the $k$-th SU as $\chi_{k}$, which can be expressed as

$$
\chi_{k}=\left|\mathbf{v}^{H} \mathbf{U}_{k} \mathbf{w}_{k}\right|^{2}-\gamma_{0}\left(\sum_{i \neq k}^{K}\left|\mathbf{v}^{H} \mathbf{U}_{k} \mathbf{w}_{i}\right|^{2}+\sigma^{2}\right), \quad k=1, \ldots, K .
$$

As discussed earlier, when the beamforming vectors are obtained, the current setting already satisfies the SINR constraint condition in (17). Therefore, $\chi_{k}$ satisfies the following constraint condition:

$$
\chi_{k} \geq \chi_{0}, \quad k=1, \ldots, K,
$$

where $\chi_{0}$ is a constant that is greater than zero. $\chi_{0}$ denotes the base lower bound of $\chi_{k}, k=1, \ldots, K$. If the base lower bound $\chi_{0}$ is increased, it is easy to know that higher $\gamma_{0}$ can be obtained and the SINR constraints in (20) are satisfied. Therefore, to satisfy the conditions in (20), we need to maximize $\chi_{0}$.

Based on the above analysis, we rewrite (P6) as

P7.1:

$$
\begin{array}{ll}
\max _{\mathbf{v}} & \min \quad \chi_{k}, \\
\text { s.t. } & \chi_{k}=\left|\mathbf{v}^{H} \mathbf{U}_{k} \mathbf{w}_{k}\right|^{2}-\gamma_{0}\left(\sum_{i \neq k}^{K}\left|\mathbf{v}^{H} \mathbf{U}_{k} \mathbf{w}_{i}\right|^{2}+\sigma^{2}\right) ; \\
& \chi_{k} \geq 0, \quad k=1, \ldots, K, \\
& \omega_{n} \in[0,2 \pi), \quad n=1, \ldots, N .
\end{array}
$$

Then, we use the SDP-based method to relax (P7.1) into an SDP problem. We have the following equations:

$$
\begin{aligned}
\mid \mathbf{v}^{H} & \left.\mathbf{U}_{k} \mathbf{w}_{k}\right|^{2} \\
& =\mathbf{v}^{H} \mathbf{U}_{k} \mathbf{w}_{k} \times\left(\mathbf{v}^{H} \mathbf{U}_{k} \mathbf{w}_{k}\right)^{H} \\
& =\mathbf{v}^{H} \mathbf{U}_{k} \mathbf{w}_{k} \mathbf{w}_{k}^{H} \mathbf{U}_{k}^{H} \mathbf{v} \\
& =\mathbf{v}^{H} \mathbf{Q}_{k, k} \mathbf{v} \\
& =\operatorname{tr}\left(\mathbf{Q}_{k, k} \mathbf{V}\right),
\end{aligned}
$$

where $\mathbf{Q}_{k, k}=\mathbf{U}_{k} \mathbf{w}_{k} \mathbf{w}_{k}^{H} \mathbf{U}_{k}^{H}$ is a determined matrix and $\mathbf{V}=\mathbf{v v}^{H}$. Then, we can rewrite $(P 7.1)$ as

P7.2:

$$
\begin{aligned}
\max _{\mathbf{V}} & \min \quad \chi_{k}, \\
\text { s.t. } \quad \chi_{k} & =\operatorname{tr}\left(\mathbf{Q}_{k, k} \mathbf{V}\right)-\gamma_{0}\left(\sum_{i=1, i \neq k}^{K} \operatorname{tr}\left(\mathbf{Q}_{k, k} \mathbf{V}\right)+\sigma^{2}\right) ; \\
\chi_{k} & \geq 0, \quad k=1, \ldots, K ; \\
\mathbf{V} & \succ 0 ; \\
\operatorname{diag}(\mathbf{V}) & =[1,1,1, \ldots, 1 .]
\end{aligned}
$$

$(P 7.2)$ is an SDP problem that can be solved using a convex optimization tool such as CVX.

By solving (P7.2), we can obtain an optimal solution for $\mathbf{V}$. However, $\mathbf{V}$ cannot be guaranteed to be of rank one. If $\mathbf{V}$ is of rank one, we can use eigenvalue decomposition to obtain optimal $\mathbf{v}$. When $\mathbf{V}$ is not of rank one, an approximate optimal solution can be obtained using the Gauss randomization method presented in [38].

3.3. Algorithm Implementation. The main steps of the optimization process are as follows: 
(1) First, we randomly set the AIRS reflection coefficient vector $\mathbf{v}_{0}$ and use $\mathbf{v}_{0}$ to optimize the beamforming vectors.

(2) Then, we use the optimized beamforming vectors to update the AIRS reflection coefficient vector $\mathbf{v}$.

(3) We use the new AIRS reflection coefficient vector $\mathbf{v}$ to update the beamforming vectors at each AP. This iterative process is repeated until the total transmission power converges.

The specific steps are given in Algorithm 1.

3.4. Extension to the Multi-AIRS Case. Now, we will extend the optimization process to the multi-AIRS case. Let there be $T$ AIRSs assisting the communication between $L$ APs and $K$ SUs. The channel from the $l$-th AP to the $t$-th AIRS is represented by $\mathbf{G}_{l t}$. The channel from the $t$-th AIRS to the $k$-th $\mathrm{SU}$ is represented by $\mathbf{h}_{t k} . \mathbf{G}_{l t}$ and $\mathbf{h}_{t k}$ are defined according to (4) and (5), respectively. The reflection coefficient matrix of the $t$-th AIRS is $\Phi_{t}(t=1,2, \ldots, T)$. The channel from the $l$-th AP to the $k$-th SU reflected by the $t$-th AIRS can be expressed as

$$
\begin{aligned}
\mathbf{c}_{l, t, k} & =\mathbf{h}_{t k} \boldsymbol{\Phi}_{t} \mathbf{G}_{l t} \\
& =\mathbf{v}_{t}^{H} \operatorname{diag}\left(\mathbf{h}_{t k}\right) \mathbf{G}_{l t} .
\end{aligned}
$$

The joint channel matrix from the $l$-th AP to the $k$-th SU can be expressed as

$$
\begin{aligned}
y_{k} & =\sum_{l=1}^{L} \sum_{t=1}^{T} \mathbf{c}_{l, t, k} \sum_{i=1}^{k} \mathbf{w}_{l i} s_{i} \\
& =\sum_{l=1}^{L} \sum_{t=1}^{T} \mathbf{v}_{t}^{H} \operatorname{diag}\left(\mathbf{h}_{t k}\right) \mathbf{G}_{l t} \sum_{i=1}^{k} \mathbf{w}_{l i} s_{i} \\
& =\sum_{l=1}^{L} \widetilde{\mathbf{v}}^{H} \operatorname{diag}\left(\widetilde{\mathbf{h}}_{k}\right) \widetilde{\mathbf{G}}_{l} \sum_{i=1}^{k} \mathbf{w}_{l i} s_{i} \\
& =\sum_{l=1}^{L} \widetilde{\mathbf{c}}_{l k} \sum_{i=1}^{k} \mathbf{w}_{l i} s_{i},
\end{aligned}
$$

where $\widetilde{\mathbf{c}}_{l k}=\widetilde{\mathbf{v}}^{H} \operatorname{diag}\left(\widetilde{\mathbf{h}}_{k}\right) \widetilde{G}_{l}, \widetilde{\mathbf{v}}^{H}=\left[\mathbf{v}_{1}^{H}, \mathbf{v}_{2}^{H}, \ldots, \mathbf{v}_{T}^{H}\right] \in \mathbb{C}^{1 \times N T}$, $\widetilde{\mathbf{h}}_{k}=\left[\mathbf{h}_{1 k}, \mathbf{h}_{2 k}, \ldots, \mathbf{h}_{T k}\right] \in \mathbb{C}^{N T \times 1}, \quad$ and $\widetilde{\mathbf{G}}_{l}=\left[\mathbf{G}_{l 1}, \mathbf{G}_{l 2}\right.$, $\left.\ldots, \mathbf{G}_{l T}\right]^{T} \in \mathbb{C}^{N T \times L M}$.

The SINR expression is similar to (8), and the objective function is similar to (9). By replacing $\mathbf{c}_{l k}$ with $\widetilde{c}_{l k}$, we can use the proposed Algorithm 1 to solve this problem as well.

\section{Simulation Results}

In this section, we present a numerical simulation of an actual communication scenario conducted to study the performance of the proposed algorithm. First, we analyse the performance of the single-AIRS case. Then, we extend to the Multi-AIRS case, and we mainly focus on the performance comparison for AIRS deployment.
4.1. Single-AIRS Case. As shown in Figure 2, three APs serve four SUs with the assistance of an AIRS. The AIRS is deployed at a fixed point $(0,75,40)$. All the APs and SUs are in the $x-o-y$ plane. The parameter settings are detailed in Table 1.

In Figure 3, we compare the performance under three different strategies:

(1) Beamforming optimization with random reflection design: In this setting, we randomly set the AIRS reflection coefficients and optimize the beamforming vectors $\mathbf{w}_{k}(k=1,2, \ldots, K)$.

(2) Zero forcing (ZF) beamforming with random reflection design: In this setting, we use the $\mathrm{ZF}$ beamforming strategy to set the beamforming vectors $\mathbf{w}_{k}(k=1,2, \ldots, K)$. In this system, we randomly set the AIRS reflection coefficients and then use the global channel to design the ZF beamforming vectors. The global channel is denoted by $\mathbf{C} \in \mathbb{C}^{K \times M L}$ and is expressed as follows:

$$
\begin{aligned}
\mathbf{C} & =\left[\begin{array}{cccc}
\mathbf{c}_{11} & \mathbf{c}_{21} & \ldots & \mathbf{c}_{L 1} \\
\mathbf{c}_{12} & \mathbf{c}_{22} & \ldots & \mathbf{c}_{L 2} \\
\vdots & \vdots & \vdots & \vdots \\
\mathbf{c}_{1 K} & \mathbf{c}_{2 K} & \ldots & \mathbf{c}_{L K}
\end{array}\right] \\
& =\left[\begin{array}{c}
\mathbf{c}_{1} \\
\mathbf{c}_{2} \\
\vdots \\
\mathbf{c}_{K}
\end{array}\right] .
\end{aligned}
$$

The pseudoinverse of $\mathbf{C}$ is

$$
\begin{aligned}
\overline{\mathbf{C}} & =\mathbf{C}^{H}\left(\mathbf{C C}^{H}\right)^{-1} \\
& =\left[\overline{\mathbf{c}}_{1}, \overline{\mathbf{c}}_{2}, \ldots, \overline{\mathbf{c}}_{K}\right] .
\end{aligned}
$$

We set the beamforming vectors as follows:

$$
\mathbf{w}_{k}=\sqrt{p_{k}} \frac{\bar{c}_{k}}{\left\|\bar{c}_{k}\right\|_{2}} .
$$

Then, we calculate the minimum transmit power $P$ that satisfies the SINR constraint. $P$ is defined as

$$
P=p_{1}+p_{2}, \ldots,+p_{k}
$$

(3) ZF beamforming with exhaustion-based AIRS reflection design: In this setting, we randomly set $\mathbf{v}$ and use the ZF beamforming method 1000 times. We choose the best performance of 1000 experiments as the output.

(4) ZF beamforming with the channel gain maximization-based AIRS reflection design: In this setting, we use ZF beamforming and a channel energy maximization-based AIRS phase optimization method to design the AIRS reflection coefficient. The main consideration is that after $\mathrm{ZF}$ beamforming, the interference between SUs has been eliminated. One 
(1) Initialize the AIRS reflection coefficient vector $\mathbf{v}_{0}$.

(2) Update the beamforming vectors $\mathbf{w}_{k}$ by solving (P4) and calculate the total transmission power.

(3) Use the updated beamforming vectors $\mathbf{w}_{k}$ to obtain $\mathbf{V}$ by solving (P7.2).

(4) If $\mathbf{V}$ is of rank one Obtain the optimal $\mathbf{v}$ via rank-one decomposition. Else Obtain the optimal $\mathbf{v}$ via Gauss randomization. End

(5) Repeat steps 2-4. If the absolute value of the difference of transmission power between the $n$-th and the $(n-1)$-th iterations is less than 0.0001 , the convergence is achieved. Then output the current $\mathbf{w}_{k}$ and $\mathbf{v}$.

\section{Algorithm 1: Joint optimization algorithm.}

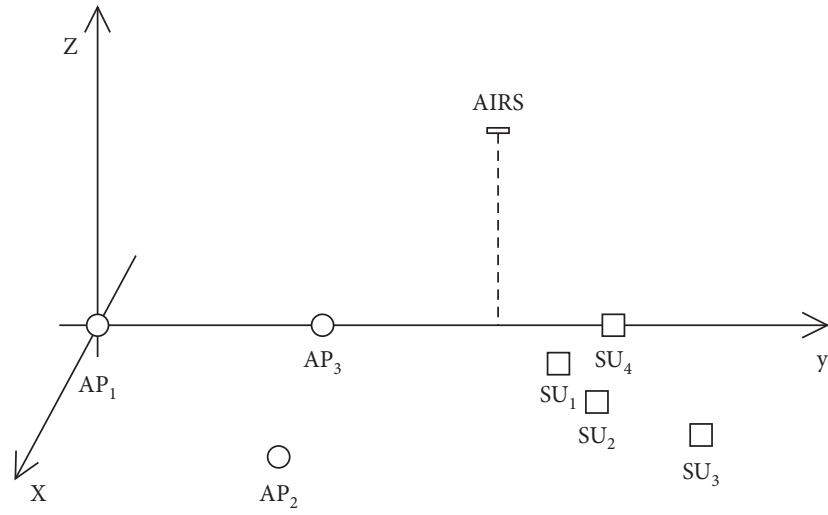

FIGURE 2: Simulation scenario of the single-AIRS case.

TABle 1: Simulation settings.

\begin{tabular}{lc}
\hline Parameter & Value \\
\hline Bandwidth & $1 \mathrm{MHz}$ \\
Number of antennas at each AP, M & 20 \\
Number of AIRS elements, $\mathrm{N}$ & 30 \\
Noise power & $-110 \mathrm{dBm}$ \\
Rice factor, $\kappa$ & 10 \\
Coordinates of $\mathrm{AP}_{1}(\mathrm{~m})$ & $(0,0,0)$ \\
Coordinates of $\mathrm{AP}_{2}(\mathrm{~m})$ & $(0,50,0)$ \\
Coordinates of $\mathrm{AP}_{3}(\mathrm{~m})$ & $(50,50,0)$ \\
Coordinates of $\mathrm{AIRS}^{(\mathrm{m})}$ & $(0,90,40)$ \\
Coordinates of $\mathrm{SU}_{1}(\mathrm{~m})$ & $(0,100,0)$ \\
Coordinates of $\mathrm{SU}_{2}(\mathrm{~m})$ & $(10,95,0)$ \\
Coordinates of $\mathrm{SU}_{3}(\mathrm{~m})$ & $(20,100,0)$ \\
Coordinates of $\mathrm{SU}_{4}(\mathrm{~m})$ & $(-10,100,0)$ \\
Antenna or element spacing, $d$ & $0.5 \lambda$ \\
\hline
\end{tabular}

method is to maximize the total channel gain so that the beamforming gain is optimal.

First, we optimize the AIRS reflection vector $\mathbf{v}$ by the channel gain maximization-based method. Then we use $\mathbf{v}$ to design the $\mathrm{ZF}$ beamforming. The total channel gain is given as:

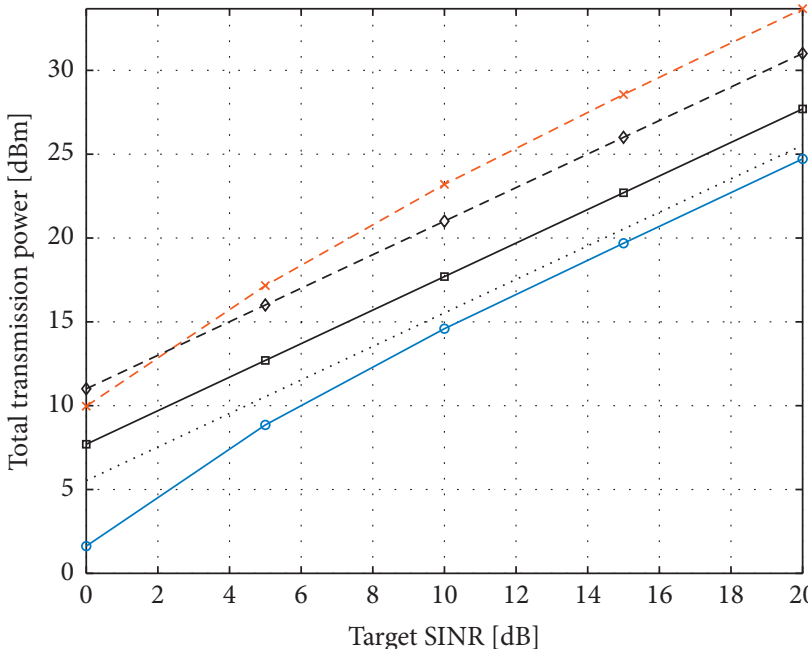
$\rightarrow$ Proposed method
$\rightarrow$ ZF beamforming with the channel gain maximization-based AIRS reflection design
-* - Beamforming optimization
with random reflection
design

..... ZF beamforming with exhaustion-based AIRS reflection design

$-\diamond-$ ZF beamforming with random reflection design

Figure 3: Performance comparison.

$$
\begin{aligned}
C & =\sum_{k=1}^{K}\left\|\mathbf{c}_{k}\right\|^{2} \\
& =\sum_{k=1}^{K} \mathbf{c}_{k} \mathbf{c}_{k}^{H} \\
& =\sum_{k=1}^{K} \sum_{l=1}^{L} \mathbf{v}^{H} \mathbf{U}_{l k} \mathbf{U}_{l k}^{H} \mathbf{v} \\
& =\sum_{k=1}^{K} \sum_{l=1}^{L} \operatorname{tr}\left(\tilde{\mathbf{U}}_{l k} \mathbf{V}\right),
\end{aligned}
$$

where $\widetilde{\mathbf{U}}_{l k}=\mathbf{U}_{l k} \mathbf{U}_{l k}^{H}$. The objective function for optimizing $\mathbf{v}$ is formulated as follows: 


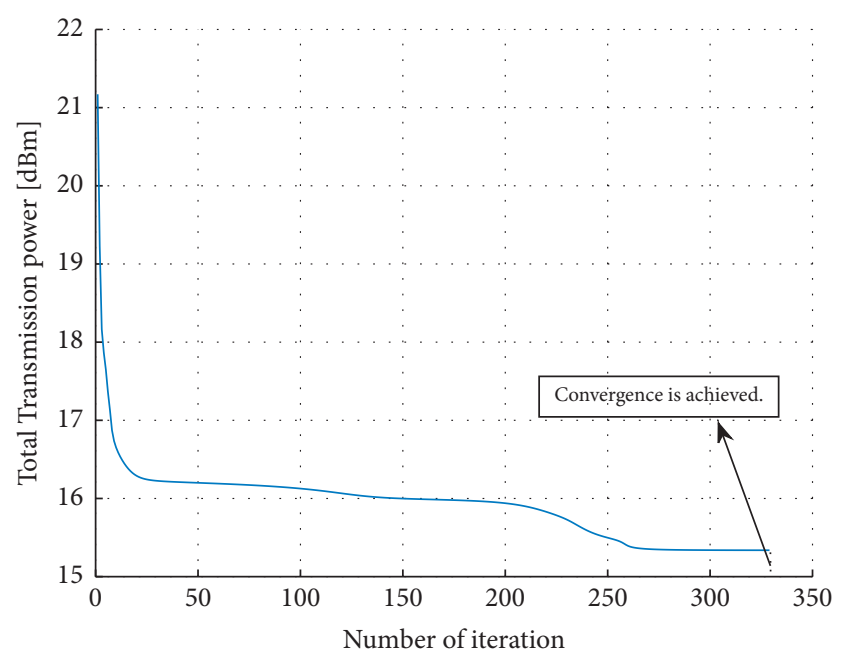

FIGURE 4: Convergence analysis.

$$
\begin{aligned}
& \max _{\mathbf{V}} \sum_{k=1}^{K} \sum_{l=1}^{L} \operatorname{tr}\left(\widetilde{\mathbf{U}}_{l k} \mathbf{V}\right), \\
& \text { s.t. } \mathbf{V}>0 \\
& \operatorname{diag}(\mathbf{V})=[1,1,1, \ldots, 1 .]
\end{aligned}
$$

Here, we use a similar approach to $[22,39]$ to solve this problem. Then the optimal $\mathbf{v}$ can be obtained. For brevity, the specific details have been omitted.

(5) Proposed method: In this setting, we use the proposed method to optimize both the beamforming vectors and the AIRS reflection coefficients.

As shown in Figure 3, By optimizing the AIRS reflection coefficients, the total transmission power can be effectively reduced. Overall, the proposed method shows better performance in saving the total transmission power.

In Figure 4, we analyze the convergence of the proposed algorithm. We set the target SINR to $10 \mathrm{~dB}$, while the other parameters are set as before. If the absolute value of the difference of transmission power between the $n$-th and the $(n-1)$-th iterations is less than 0.0001 , convergence is achieved. As seen from Figure 4, through iteration, the total transmission power is significantly reduced and finally converges to a value. The proposed method has strict convergence.

In Figure 5, we study the impact of the AIRS scale on the algorithm performance. We set the number of AIRS elements $N$ to $10,20,30,40,50$, and 60 , and the target SINR is set to $10 \mathrm{~dB}$. Figure 5 shows that as the number of AIRS elements increases, the total transmission power decreases if both the beamforming and AIRS reflection are optimized, indicating that more AIRS elements can be deployed to further reduce the transmission power with the proposed method.

4.2. Multi-AIRS Case. Then, we study the multi-AIRS case. We mainly analyze the impact of the AIRS deployment

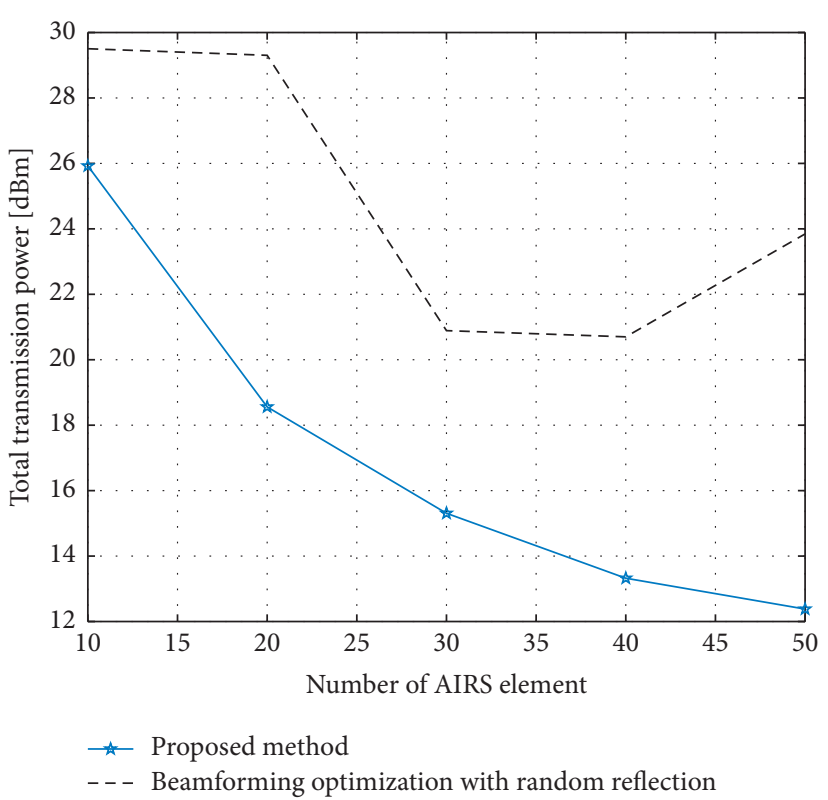

FIgURE 5: Impact of the number of AIRS elements.

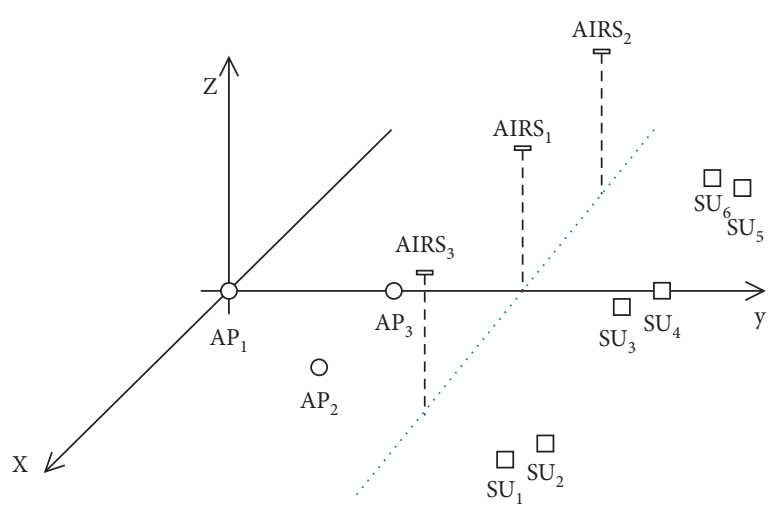

FIgURE 6: Simulation scene of multi-AIRS case.

strategy on performance. Assume that there are six SUs. As shown in Figure 6, the SUs are distributed in three communities. We aim to compare the performance of centralized and distributed AIRS deployment.

For centralized AIRS deployment, we use one AIRS to assist communication. The number of AIRS elements is set as 30 . The AIRS is deployed at $(0,90,40)$. For distributed AIRS deployment, we use three AIRSs to assist communication. To be fair, the number of elements of each AIRS is set as 10 . The AIRSs are deployed at $(0,90,40),(60,90,40)$, and $(-60,90,40)$. The coordinates are detailed in Table 2 . The other settings are the same as the single-AIRS case.

As we can see from Figure 7, the distributed AIRS deployment shows a better performance in reducing the total transmission power. One reasonable explanation is that distributed AIRS deployment can provide more flexible deployment according to the location of the SUs so that the AIRS can be deployed closer to the SUs to better assist communication. Another reason is that distributed AIRS 
TABLE 2: Coordinate settings.

\begin{tabular}{lc}
\hline Parameter & Value \\
\hline Coordinates of $\mathrm{AP}_{1}(\mathrm{~m})$ & $(0,0,0)$ \\
Coordinates of $\mathrm{AP}_{2}(\mathrm{~m})$ & $(50,50,0)$ \\
Coordinates of $\mathrm{AP}_{3}(\mathrm{~m})$ & $(0,50,0)$ \\
Coordinates of $\mathrm{AIRS}_{1}(\mathrm{~m})$ & $(0,90,40)$ \\
Coordinates of $\mathrm{AIRS}_{2}(\mathrm{~m})$ & $(-60,90,40)$ \\
Coordinates of $\mathrm{AIRS}_{3}(\mathrm{~m})$ & $(60,90,40)$ \\
Coordinates of $\mathrm{SU}_{1}(\mathrm{~m})$ & $(60,100,0)$ \\
Coordinates of $\mathrm{SU}_{2}(\mathrm{~m})$ & $(60,95,0)$ \\
Coordinates of $\mathrm{SU}_{3}(\mathrm{~m})$ & $(0,105,0)$ \\
Coordinates of $\mathrm{SU}_{4}(\mathrm{~m})$ & $(5,100,0)$ \\
Coordinates of $\mathrm{SU}_{5}(\mathrm{~m})$ & $(-60,100,0)$ \\
Coordinates of $\mathrm{SU}_{6}(\mathrm{~m})$ & $(-60,105,0)$ \\
\hline
\end{tabular}

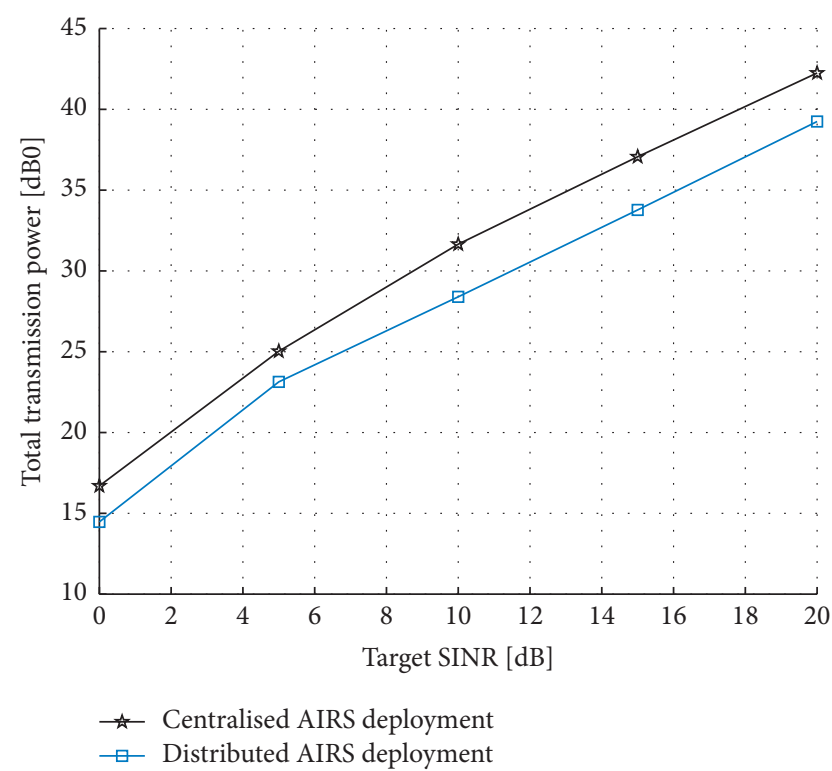

FIGURE 7: Impact of the AIRS deployment strategy.

deployment can provide a relatively rich scattering environment because the reflection is increased. The rich scattering environment can reduce the correlation among the channels of different SUs; thus, the interference between different SUs can be suppressed.

\section{Conclusion}

In this paper, we study a power minimization problem in an AIRS-aided CF massive MIMO WSN system. We propose a joint optimization method to design the beamforming vectors and the AIRS reflection coefficients. Simulation results illustrate the good performance of the proposed method. This work deserves further study in the following aspects. In this paper, we simply assume that perfect channel state information (CSI) is obtained. However, channel estimation is challenging in this system. Therefore, in our future work, we will consider a suitable channel estimation method for this system. Moreover, AIRS deployment requires the location of SUs. AIRS-aided user positioning also deserves further study.

\section{Data Availability}

No data were used to support this study.

\section{Conflicts of Interest}

The authors declare that they have no conflicts of interest.

\section{Acknowledgments}

This work was supported in part by the National Natural Science Foundation of China under Grant 62071485 and Grant 61901519, in part by the Basic Research Project of Jiangsu Province under Grant BK 20192002, and by the Natural Science Foundation of Jiangsu Province under Grant BK 20201334 and Grant BK 20181335.

\section{References}

[1] S. Dang, O. Amin, B. Shihada, and M. Alouini, "What should 6G be?" Nature Electronics, vol. 3, no. 1, pp. 20-29, 2020.

[2] Z. Lv and N. Kumar, "Software defined solutions for sensors in 6G/IoE," Computer Communications, vol. 153, pp. 42-47, 2020.

[3] V. Ziegler, H. Viswanathan, H. Flinck, M. Hoffmann, V. Raisanen, and K. Hatonen, " $6 \mathrm{G}$ architecture to connect the worlds," IEEE Access, vol. 8, Article ID 173508, 2020.

[4] S. Wan, J. Hu, C. Chen, A. Jolfaei, S. Mumtaz, and Q. Pei, "Fair-hierarchical scheduling for diversified services in space, air and ground for 6G-dense Internet of things," IEEE Transactions on Network Science and Engineering, 2020.

[5] Y. Pei, S. Miao, and F. Li, Challenges and Opportunities for Terrestrial Network Operators in the Air-Space-Ground Integrated Network, in Proceedings of the 2020 IEEE Intl Conf on Parallel \& Distributed Processing with Applications, Big Data \& Cloud Computing, Sustainable Computing \& Communications, Social Computing \& Networking (ISPA/BDCloud/ SocialCom/SustainCom), pp. 1400-1404, Exeter, United Kingdom, December 2020.

[6] H. Huang, S. Hu, T. Yang, and C. W. Yuan, "Full duplex nonorthogonal multiple access with layers-based optimized mobile relays subsets algorithm in B5G/6G ubiquitous networks," IEEE Internet of Things Journal, 2020.

[7] Q. Wu and R. Zhang, "Towards smart and reconfigurable environment: intelligent reflecting surface aided wireless network," IEEE Communications Magazine, vol. 58, no. 1, pp. 106-112, 2020.

[8] E. Basar, M. Di Renzo, J. De Rosny, M. Debbah, M.-S. Alouini, and R. Zhang, "Wireless communications through reconfigurable intelligent surfaces," IEEE Access, vol. 7, Article ID 116753, 2019.

[9] et al., "Smart radio environments empowered by reconfigurable AI meta-surfaces: an idea whose time has come," EURASIP Journal on Wireless Communications and Networking, vol. 2019, no. 1, 2019.

[10] M. Di Renzo, A. Zappone, M. Debbah et al., "Smart Radio Environmentsempowered by Reconfigurable Intelligent Surfaces: How it Works, State of Research, and Road ahead," 2020. 
[11] et al., "Reconfigurable intelligent surfaces vs. Relaying: differences, similarities, and performance comparison," IEEE Open Journal of the Communications Society, vol. 1, pp. 798-807, 2020.

[12] S. Hu, F. Rusek, and O. Edfors, "Beyond massive MIMO: the potential of data transmission with large intelligent surfaces," IEEE Transactions on Signal Processing, vol. 66, no. 10, pp. 2746-2758, 2018.

[13] Q. Wu and R. Zhang, "Intelligent Reflecting Surface Enhanced Wireless Network: Joint Active and Passive Beamforming Design," in Proceedings of the 2018 IEEE Global Communications Conference (GLOBECOM), pp. 1-6, Abu Dhabi, United Arab Emirates, December 2018.

[14] Q. Wu, S. Zhang, B. Zheng, C. You, and R. Zhang, "Intelligent reflecting surface-aided wireless communications: a tutorial," IEEE Transactions on Communications, vol. 69, no. 5, pp. 3313-3351, 2021.

[15] E. G. Larsson, O. Edfors, F. Tufvesson, and T. L. Marzetta, "Massive MIMO for next generation wireless systems," IEEE Communications Magazine, vol. 52, no. 2, pp. 186-195, 2014.

[16] J. G. Andrews, S. Buzzi, W. Choi et al., "What will 5G Be?" IEEE Journal on Selected Areas in Communications, vol. 32, no. 6, pp. 1065-1082, 2014.

[17] H. Q. Ngo, A. Ashikhmin, H. Yang, E. G. Larsson, and T. L. Marzetta, "Cell-free massive MIMO versus small cells," IEEE Transactions on Wireless Communications, vol. 16, no. 3, pp. 1834-1850, 2017.

[18] E. Nayebi, A. Ashikhmin, T. L. Marzetta, H. Yang, and B. D. Rao, "Precoding and power optimization in cell-free massive MIMO systems," IEEE Transactions on Wireless Communications, vol. 16, no. 7, 2017.

[19] H. Lu, Y. Zeng, S. Jin, and R. Zhang, "Enabling Panoramic Full-Angle Reflection via Aerial Intelligent Reflecting Surface," in Proceedings of the 2020 IEEE International Conference On Communications Workshops (ICC Workshops), pp. 1-6, Dublin, Ireland, June 2020.

[20] H. Lu, Y. Zeng, S. Jin, and R. Zhang, "Aerial intelligent reflecting surface: joint placement and passive beamforming design with 3D beam flattening," IEEE Transactions on Wireless Communications, vol. 20, no. 7, pp. 4128-4143, 2021.

[21] X. Yu, D. Xu, and R. Schober, "Miso Wireless Communication Systems Via Intelligent Reflecting Surfaces: (Invited Paper)," in Proceedings of the 2019 IEEE/CIC International Conference On Communications In China (ICCC), pp. 735-740, Changchun, China, August 2019.

[22] Q. Wu and R. Zhang, "Intelligent reflecting surface enhanced wireless network via joint active and passive beamforming," IEEE Transactions on Wireless Communications, vol. 18, no. 11, pp. 5394-5409, 2019.

[23] S. Zhang and R. Zhang, "Capacity characterization for intelligent reflecting surface aided MIMO communication," IEEE Journal on Selected Areas in Communications, vol. 38, no. 8, pp. 1823-1838, 2020.

[24] C. Pan, H. Ren, K. Wang et al., "Multicell MIMO communications relying on intelligent reflecting surfaces," IEEE Transactions on Wireless Communications, vol. 19, no. 8, pp. 5218-5233, 2020.

[25] Y. Zhang, B. Di, H. Zhang et al., "Beyond cell-free MIMO: energy efficient reconfigurable intelligent surface aided cellfree MIMO communications," IEEE Transactions on Cognitive Communications and Networking, vol. 7, no. 2, pp. 412426, 2021.

[26] Z. Zhang and L. Dai, "Capacity improvement in wideband reconfigurable intelligent surface-aided cell-free network," in
Proceedings of the 2020 IEEE 21st International Workshop On Signal Processing Advances In Wireless Communications (SPAWC), pp. 1-5, Atlanta, GA, USA, May 2020.

[27] Y. Zhang, B. Di, H. Zhang, J. Lin, Y. Li, and L. Song, "Reconfigurable intelligent surface aided cell-free MIMO communications," IEEE Wireless Communications Letters, vol. 10, no. 4, pp. 775-779, 2021.

[28] S. Huang, Y. Ye, M. Xiao, H. V. Poor, and M. Skoglund, "Decentralized beamforming design for intelligent reflecting surface-enhanced cell-free networks," IEEE Wireless Communications Letters, vol. 10, no. 3, pp. 673-677, 2021.

[29] C. Guo, Y. Cui, F. Yang, and L. Ding, "Outage probability analysis and minimization in intelligent reflecting surfaceassisted MISO systems," IEEE Communications Letters, vol. 24, no. 7, pp. 1563-1567, 2020.

[30] C. You and R. Zhang, "3D trajectory optimization in rician fading for UAV-enabled data harvesting," IEEE Transactions on Wireless Communications, vol. 18, no. 6, pp. 3192-3207, 2019.

[31] Y. Han, W. Tang, S. Jin, C.-K. Wen, and X. Ma, "Large intelligent surface-assisted wireless communication exploiting statistical CSI," IEEE Transactions on Vehicular Technology, vol. 68, no. 8, pp. 8238-8242, 2019.

[32] Z.-Q. He and X. Yuan, "Cascaded channel estimation for large intelligent metasurface assisted massive MIMO," IEEE Wireless Communications Letters, vol. 9, no. 2, pp. 210-214, 2020.

[33] H. Guo, Y. Liang, J. Chen, and E. G. Larsson, "Weighted sumrate maximization for reconfigurable intelligent surface aided wireless networks," IEEE Transactions on Wireless Communications, vol. 19, no. 5, pp. 3064-3076, 2020.

[34] Y. Zeng, Q. Wu, and R. Zhang, "Accessing from the sky: a tutorial on UAV communications for 5G and beyond," Proceedings of the IEEE, vol. 107, no. 12, pp. 2327-2375, 2019.

[35] X. Lin, V. Yajnanarayana, S. D. Muruganathan et al., "The sky is not the limit: LTE for unmanned aerial vehicles," IEEE Communications Magazine, vol. 56, no. 4, pp. 204-210, 2018.

[36] M. Grant and S. Boyd, "CVX: MATLAB Software for Disciplined Convex Programming," 2016, http://cvxr.com/cvx.

[37] M. Bengtsson and B. Ottersten, "Optimal and suboptimal transmit beamforming," Handbook Of Antennas In Wireless Communications, CRC Press, Boco Raton, FL, USA, 2001.

[38] Y. Zhao, B. Clerckx, and Z. Feng, "Intelligent reflecting surface-aided swipt: joint waveform, active and passive beamforming design," 2020, https://arxiv.org/abs/2012.05646.

[39] W. Yan, X. Yuan, and X. Kuai, "Passive beamforming and information transfer via large intelligent surface," IEEE Wireless Communications Letters, vol. 9, no. 4, pp. 533-537, 2020. 